\title{
Crises humanitaires, santé des réfugiés et des déplacés : un cadre analytique
}

Danièle Laliberté

\section{(2) OpenEdition}

1 Journals

Édition électronique

URL : https://journals.openedition.org/remi/4207

DOI : $10.4000 /$ remi. 4207

ISSN : 1777-5418

Éditeur

Université de Poitiers

Édition imprimée

Date de publication : 1 décembre 2007

Pagination : 85-96

ISBN : 978-2-911627-47-4

ISSN : 0765-0752

Référence électronique

Danièle Laliberté, "Crises humanitaires, santé des réfugiés et des déplacés : un cadre analytique », Revue européenne des migrations internationales [En ligne], vol. $23-n^{\circ} 3$ | 2007, mis en ligne le 01 décembre 2010, consulté le 14 avril 2022. URL : http://journals.openedition.org/remi/4207 ; DOI : https://doi.org/10.4000/remi.4207

Ce document a été généré automatiquement le 14 avril 2022.

(c) Université de Poitiers 


\title{
Crises humanitaires, santé des réfugiés et des déplacés : un cadre analytique
}

\author{
Danièle Laliberté
}

1 Dans un contexte de crise humanitaire, la migration de refuge constitue une stratégie de survie pour certains segments de la population. De multiples phénomènes humains, climatiques et géophysiques, amènent alors des individus, des familles et même des communautés entières à effectuer une migration forcée. Il y a crise humanitaire quand les situations d'urgence déclenchées par des conflits armés, des inondations, des tremblements de terre, des éruptions volcaniques, des raz-de-marées et/ou des sécheresses désorganisent la vie de collectivités dans des proportions exceptionnelles, constituent une menace pour la vie d'un grand nombre de personnes ${ }^{1}$ et nécessitent des mesures extraordinaires pour assurer la survie, les soins et la protection, parce que les mécanismes de soutien existants ne permettent pas d'y faire face.

2 Ce texte propose une réflexion théorique sur les enjeux de santé reliés aux migrations de refuge, fondée sur une revue d'études empiriques et d'observations effectuées dans le cadre d'interventions humanitaires en Afrique. Les principaux problèmes de santé associés aux crises humanitaires et la réponse déployée par le régime humanitaire sont présentés, pour ensuite proposer un cadre analytique tout en dégageant quelques pistes de réflexion pour des études empiriques visant à mesurer l'effet de la migration forcée sur l'état de santé.

\section{Crise humanitaire : santé, morbidité et mortalité}

3 La santé des populations, plus particulièrement des migrants, est préoccupante dans un contexte de crise humanitaire. Si les catastrophes naturelles et humaines ont des conséquences souvent similaires sur la santé, par ailleurs les situations engendrées par l'action humaine résultent parfois d'une volonté délibérée d'affaiblir une population et 
sont alors beaucoup plus dévastatrices (Lautze, Leaning, Raven-Roberts, Kent et Mazutana, $2004: 2$ 139).

Certains problèmes de santé sont fréquemment observés lors des crises humanitaires : blessures, rougeole, maladies diarrhéiques (choléra, dysenterie...), infections respiratoires sévères, paludisme. Des épidémies telles que la méningite, la fièvre jaune, l'hépatite virale et la typhoïde se manifestent dans ces situations qui par ailleurs favorisent l'apparition de problèmes psychiatriques et psychosociaux. Dans certains camps de réfugiés, les maladies diarrhéiques constituent plus de $40 \%$ des décès, dont plus de $80 \%$ des cas concernent des enfants âgés de moins de deux ans (Connolly, Gayer, Ryan, Salama, Spiegel et Heymann, 2004 : 1974). La mauvaise qualité de l'eau, le manque d'hygiène et la surpopulation seraient en grande partie responsables de cette situation. Les conditions alimentaires renforcent l'effet de ces morbidités, alors que la malnutrition et les carences nutritionnelles sont fortement associées aux risques d'infection et corrélées avec la mortalité. Le dysfonctionnement des systèmes de santé, forcés d'interrompre les programmes de lutte contre la tuberculose, provoque la recrudescence soudaine de la maladie, également renforcée par la malnutrition, les mauvaises conditions d'hébergement, mais aussi par la densité et la mobilité de la population (Connolly, Gayer, Ryan, Salama, Spiegel et Heymann, $2004: 1976)$. On a ainsi relevé une hausse de l'endémie tuberculeuse dans les deux plus grandes villes de Congo Brazzaville (Brazzaville et Pointe-Noire), dont la population s'est respectivement accrue de $30 \%$ et de $50 \%$ par les migrations rurales-urbaines de personnes déplacées durant la guerre de 1997 à 1999 (M'Boussa, Yokolo, Pereire et Ebata-Mongo, 2002 : 1). En 2000, on a constaté que le nombre de tuberculeux avait doublé et que les abandons thérapeutiques étaient passés de $20 \%$ à $26 \%$.

Lors des crises humanitaires, outre les blessures et les morts violentes résultant des combats armés, de la pratique de la torture et du recours au génocide, l'absence de contrôle social, les comportements à risque tels que ceux des militaires, les viols et le recours à la prostitution, favorisent le développement des épidémies, en particulier du $\mathrm{VIH} /$ sida, le tout dans des situations caractérisées par le manque de préservatifs, l'usage de sang infecté lors de transfusions sanguines, l'absence d'examens sanguins et de services en matière de sexualité et de santé périnatale. Si on suppose généralement que le niveau atteint par le $\mathrm{VIH} /$ sida est plus élevé dans de tels contextes, il dépend par ailleurs du taux de prévalence avant la crise. Ainsi, en Sierra Leone, le taux d'infection est plus bas que dans certains pays non marqués par un conflit (Connolly, Gayer, Ryan, Salama, Spiegel et Heymann, 2004 : 1974). En 1996, le taux de prévalence du VIH dans les armées angolaise et zimbabwéenne était estimé à $50 \%$, comparativement à $10 \%$ chez la population civile, alors que l'armée ougandaise avait une séro-prévalence de 35-45\%, et que le taux de mortalité dû au VIH/sida était de $34 \%$ dans l'armée congolaise (Kinnah, 1996 dans Williamson, 2004 : 13). Des Rwandaises ayant survécu à un viol ont affiché un taux de séro-prévalence de $17 \%$ comparativement à $11 \%$ pour la population générale (McGinn, 2000 dans Williamson, 2004: 23). Alors que le taux de prévalence avant la guerre au Rwanda était de $1 \%$ dans les zones rurales et de $10 \%$ dans les zones urbaines, après la guerre il atteignait $11 \%$ dans les deux cas (Bayard, $2004: 7)$. 


\section{Régime humanitaire}

6 Le concept de gouvernance humanitaire a été introduit pour caractériser le recours au Droit international humanitaire et aux divers instruments juridiques relatifs aux droits humains pour gouverner le comportement des organisations gouvernementales et non gouvernementales dans les zones de conflit (Lautze, Leaning, Raven-Roberts, Kent et Mazurana, 2004). À titre de rappel, sur le plan juridique, les réfugiés sont spécifiquement considérés dans le cadre du Droit des réfugiés à travers la Convention (1951) et le Protocole (1967) des Nations unies², alors que le régime appliqué aux personnes déplacées est en fait constitué par un ensemble d'instruments relevant du Droit international humanitaire (DIH), du Droit international des Droits de l'Homme (DIDH), du Droit interne et des Principes directeurs relatifs au déplacement de personnes à l'intérieur de leur propre pays formulés par le Haut Commissariat des Nations unies aux Droits de l'Homme (1998). Les droits des réfugiés et personnes déplacées par rapport à la santé sont reconnus par le Droit international humanitaire ${ }^{3}$, le Droit des réfugiés ${ }^{4}$ et le Droit humanitaire ${ }^{5}$ (Bayard, 2004). L'accomplissement du droit à la santé dépend aussi du respect de certains droits fondamentaux couverts par d'autres instruments juridiques ${ }^{6}$. Rappelons enfin que la santé de la reproduction a été reconnue comme un droit humain fondamental à la Conférence internationale sur la Population et le Développement de 1994. La Déclaration universelle des Droits de l'Homme adoptée par la majorité des pays membres l'ONU en 1949 a consacré le droit d'ingérence international «immatériel» dans les questions relevant de la responsabilité des États, alors que des résolutions de l'ONU ont ensuite défini les principes de l'assistance humanitaire et le droit de secourir les victimes de conflits selon une perspective transfrontalière (St-Pierre, 2002: 50). Ainsi, les principes sur les personnes déplacées visent à prévenir les déplacements forcés et à garantir le droit et la protection de ces migrants durant le déplacement, leur retour et leur réinsertion (International Committee of the Red Cross, 1998). En vertu de ce cadre, les personnes déplacées ont les mêmes droits que les autres membres de la population, les autorités étatiques étant tenues de leur offrir protection et assistance et d'autoriser les organismes humanitaires à les assister.

7 Dans le cas de conflits armés cependant, pour des raisons d'ordre politique l'intervention humanitaire ne s'exerce pas dans les mêmes conditions pour les réfugiés et les personnes déplacées. En particulier, l'accès à l'aide humanitaire est souvent compromis par les combats et les pressions exercées par les États; elle fait l'objet de négociations n'aboutissant pas toujours à la préservation des intérêts de ces populations vulnérables. Les populations civiles sont d'ailleurs systématiquement utilisées à la fois comme enjeu et objectif militaires (Conoir, 2002 : 147). Lors d'une catastrophe d'origine naturelle ou humaine, l'aide humanitaire vise à prévenir et à diminuer la morbidité et la mortalité excessives et à promouvoir un retour à la normale (Le Projet Sphère, $2004: 302$ ). Elle est adaptée à la nature de la situation et elle évolue en fonction des diverses phases traversées, de l'émergence de la crise à sa résolution. Ainsi, les guerres, les tremblements de terre et les inondations, pour ne nommer que ceux-là, engendrent des types de morbidité et de mortalité d'intensité et de tendances variables.

8 Deux principaux modèles globaux d'assistance humanitaire ont été élaborés, fondés sur une stratégie d'intervention arrimée aux systèmes du pays d'accueil, ou au contraire 
sur une stratégie d'intervention indépendante. L'approche intégrée est adoptée quand les structures et ressources locales sont en mesure de répondre à la fois aux besoins des réfugiés, des personnes déplacées et de la population locale. L'intervention humanitaire s'appuie alors sur les acquis locaux tout en contribuant à les renforcer afin de les rendre également disponibles aux nationaux et aux migrants. Cette solution comporte des avantages en termes de durabilité et d'équité. Une telle stratégie a été déployée en Guinée et a eu un impact positif sur la santé de la population d'accueil (Van Damme, De Brouwere, Boelaert et Van Lerberghe, 1998). En effet, la présence de réfugiés et l'existence du programme d'assistance aux réfugiés sont associées à une hausse significative du taux d'interventions obstétricales. Par ailleurs, l'étude reconnaît l'effet conjugué d'autres variables ayant pu simultanément favoriser l'amélioration de l'accès aux services de santé.

Si en arrivant dans le pays d'accueil les réfugiés ont souvent recours aux services de santé locaux, les interventions indépendantes s'imposent lorsque les structures et ressources locales sont inexistantes, inaccessibles ou incapables de répondre à la demande. Il s'agit alors de créer des structures et une offre de services à l'intérieur des camps. Les réfugiés et personnes déplacées sont ciblés par l'aide humanitaire. Malheureusement, des écarts entre l'état de santé des réfugiés, des personnes déplacées et de la population locale ont été observés à maintes reprises quand ce modèle était appliqué. À titre d'exemple, une recherche effectuée en Ouganda en 1999-2002 a démontré que la mortalité maternelle de la population d'accueil était de deux à cinq fois plus élevée que celle des réfugiés, qui avaient un meilleur accès géographique et financier à des soins obstétricaux de qualité (Orach et De Brouwere, 2004). Une fois la crise stabilisée, l'élargissement des services de santé de la reproduction est recommandé afin de répondre aux besoins à long terme des réfugiés tout en faisant participer les communautés locales; si les réfugiés sont dispersés dans la population d'accueil, l'option préconisée est de consolider le système public existant (Creel, 2002). La prévention et le contrôle des maladies transmissibles, la gestion et la surveillance des cas constituent les composantes de base de la réponse humanitaire permettant de réduire la morbidité et la mortalité dans les populations affectées par les guerres (Connolly, Gayer, Ryan, Spiegel et Heymann, $2004: 1978$ ). Après avoir adopté en 1993 une résolution préconisant le développement de mesures sur la protection des réfugiés et la violence sexuelle, en 1995 le HCR a élaboré un protocole pour prévenir et traiter les cas de violence sexuelle dans les camps. En collaboration avec d'autres intervenants, il définit des services initiaux minimums (MISP ou Minimum Initial Service Package) visant essentiellement à diminuer les taux de mortalité maternelle et infantile (HCR, 2001).

10 Les services en matière de santé de la reproduction diffèrent selon la phase traversée par les bénéficiaires durant la crise humanitaire. Ainsi, les services initiaux minimums sont fournis dans le cadre de secours d'urgence. Puis, à long terme, il y a une extension des services selon une approche communautaire. Le "paquet minimum " en période d'urgence vise à prévenir et à gérer les conséquences de la violence sexuelle (contraception d'urgence...) et à planifier des services de santé de la reproduction intégrés aux soins de santé primaire. Ils espèrent contribuer à la réduction de la transmission du VIH/sida (conseils aux agents de santé et distribution de condoms...). De même, on privilégie la prévention de la morbidité et de la mortalité néo-natales et maternelles (distribution de trousses d'accouchement...). Par ailleurs, durant la phase de stabilisation on élargit les services de santé maternelle et infantile pour réduire les 
maladies et décès reliés à la grossesse et à l'accouchement. Les services de planification familiale sont alors offerts aux réfugiés et personnes déplacées (IEC, planification et accès aux moyens contraceptifs). On vise aussi la réduction, la prévention et le traitement des infections sexuellement transmissibles en particulier du VIH/sida. La prévention de la violence sexuelle, la protection des individus et leur assistance à travers soins médicaux et psychologiques sont privilégiées. Enfin, des mesures sont préconisées pour favoriser la santé de la reproduction dans les camps de réfugiés et de personnes déplacées. On favorise l'amélioration de l'adéquation de l'aide humanitaire par l'orientation sexospécifique des interventions et l'augmentation du recours aux services de santé de la reproduction par le recrutement de personnel féminin. On a constaté la diminution de la prostitution par l'application de mécanismes de distribution et de gestion égalitaires. De plus, on favorise le renforcement du dépistage $\mathrm{du} \mathrm{VIH} /$ sida par la confidentialité des tests et la sensibilisation des familles et des communautés. Cependant, le recours à l'assistance humanitaire pour répondre aux besoins de santé est une solution temporaire en attendant la réhabilitation et la reconstruction des systèmes socio-économiques. Il ne faut cependant pas perdre de vue que les réfugiés et personnes déplacées développent aussi leurs propres mécanismes de survie aux niveaux familial et communautaire.

\section{Cadre d'analyse}

11 Un essai de modélisation de l'effet des migrations forcées sur la santé des populations est présenté aux figures 1 et 2 . La première figure identifie les niveaux, domaines et champs d'analyse, pour lesquelles les variables et modalités seraient éventuellement à définir. La seconde figure présente les liens existants entre les niveaux, domaines et champs. La dimension temporelle des crises humanitaires et de la dynamique démographique sont omniprésentes dans l'ensemble des composantes du modèle. Au cœur de l'analyse, la santé est envisagée en vertu d'un continuum passant de l'état de bonne santé à la maladie puis à la mort. Elle est conceptualisée dans le cadre de l'ensemble du régime démographique, " un sous-ensemble social qui s'occupe d'une partie de la production et de la reproduction des êtres humains. Plus spécifiquement, il comprend tous les éléments dits démographiques qui ajoutent des membres à la société ou en retranchent " (Gregory et Piché, 1985: 76). Il s'agit d'un système d'entrées (naissances et immigration) et de sorties (décès et émigration). Dans la problématique qui nous préoccupe, les liens existants entre la stratégie migratoire (rester sur place, exil ou déplacement interne) et l'état de santé constituent la trame de fond de l'analyse, qui par ailleurs incorpore aussi des aspects liés à la vie génésique, les formes de conjugalité et la mobilité conjugale, en raison de leur importance dans la constitution et la dislocation de la famille. Les structures familiales jouent un rôle déterminant dans la reproduction des populations et les transformations du régime démographique des sociétés africaines (Baya et Laliberté, 2007). Elles occupent une place stratégique pour la compréhension et l'explication des phénomènes démographiques et de leur dynamique (Tabutin et Bartiaux, 1986). Lors d'une crise humanitaire, la migration qui assure la survie immédiate des membres de l'unité familiale est susceptible d'entraîner leur dispersion et donc d'affecter l'ensemble du régime démographique. Outre le fait que l'expérience d'une première migration forcée se traduit par une forte probabilité de migrer à nouveau, la migration forcée augmente le risque de divorce des personnes 
déplacées. C'est ce que nous avons observé dans une étude réalisée au Tchad en 1993-1994 (Laliberté, Laplante et Piché, 2003).

12 À un niveau d'analyse intermédiaire se situe le mode de production, composé des facteurs reliés à l'habitat, au système de santé, aux conditions socio-économiques et à la sécurité alimentaire. On suppose que durant une crise humanitaire le mode de production d'une population se caractérise par une combinaison spécifique des forces de production $^{7}$ et des relations de production sociales et techniques ${ }^{8}$. La sécurité alimentaire est compromise par le ralentissement de la production et la dislocation des réseaux de distribution des aliments. On assiste à la mutation des fondements de la sécurité sociale et à l'effritement des filets de sécurité traditionnels.

13 Au niveau sociétal, le cadre d'analyse prend en compte la gouvernance, le régime humanitaire, les environnements physique et humain. Ainsi, le modèle de gouvernance et les politiques qui en découlent déterminent en partie le mode de production et se répercutent sur le mode de reproduction, en particulier sur le régime démographique et donc sur l'état de santé. La gestion des catastrophes naturelles et humaines qui affectent l'environnement des populations est tributaire de la gouvernance et des politiques nationales et internationales. Elle dépend également du régime humanitaire élaboré conjointement avec la communauté internationale, en autres du cadre juridique, des stratégies d'interventions et des programmes mis en place. L'application de ce régime s'effectue dans les limites imposées par les enjeux politiques reliés à la nature de la catastrophe. Ainsi, tel que mentionné plus haut, dans le cas des conflits armés l'assistance des personnes déplacées entre parfois en contradiction avec les intérêts politiques de leur État et de certaines factions politico-militaires.

Figure 1 : Description des niveaux, domaines et champs d'analyse

\begin{tabular}{|l|l|}
\hline NIVEAUX \& DOMAINES & CHAMPS \\
\hline NIVEAU MACRO-ENVIRONNEMENTAL \\
\hline Environnement humain & Catastrophe humaine (confilt armé, crise socio-politique, famine) \\
\hline Environnement physique & $\begin{array}{l}\text { Catastrophe naturelle (inondation, tremblement de terre, éruption } \\
\text { volcanique, raz-de-marée, sécheresse) }\end{array}$ \\
\hline NIVEAU MACRO-SOCIÉTAL & $\begin{array}{l}\text { Politiques d'hygiène et d'assainissement, de santé, socio-économiques } \\
\text { et agricoles }\end{array}$ \\
\hline Gouvernance & $\begin{array}{l}\text { Cadre juridique (international, régional et national) } \\
\text { Stratégie d'intervention (arrimée / séparée) }\end{array}$ \\
\hline Régime humanitaire & \\
\hline \hline NIVEAU INTERMÉDIAIRE &
\end{tabular}




\begin{tabular}{|c|c|}
\hline Mode de production & $\begin{array}{l}\text { Habitat (eaux de boisson et usées, déchets, camp / établissement } \\
\text { spontané) } \\
\text { Système de santé (promotion, prévention, soins, surveillance) } \\
\text { Sécurité alimentaire (production, distribution, accès) } \\
\text { Conditions socio-économiques (forces de production, relations de } \\
\text { production, sécurité sociale) }\end{array}$ \\
\hline \multicolumn{2}{|c|}{ MODE DE REPRODUCTION (RÉGIME DÉMOGRAPHIQUE ET STRUCTURES FAMILIALES) } \\
\hline \multicolumn{2}{|l|}{ Régime démographique } \\
\hline Migration & $\begin{array}{l}\text { Stratégie migratoire (rester sur place / refuge / déplacement interne) } \\
\text { Phase du refuge ou du déplacement (installation / réinstallation / } \\
\text { retour) }\end{array}$ \\
\hline Fécondité \& nuptialité & $\begin{array}{l}\text { Naissances vivantes (nombre, intervalle, première naissance) } \\
\text { Formes de conjugalité (mariage ou union libre monogamique ou } \\
\text { polygamique) } \\
\text { Mobilité conjugale (veuvage / séparation / divorce, remariage) }\end{array}$ \\
\hline État de santé & $\begin{array}{l}\text { En bonne santé / malade (maladies parasitaires et infectieuses dont le } \\
\text { VIH, agressions, noyade, feu fumée et flamme, carences } \\
\text { nutritionnelles, troubles mentaux et du comportement, grossesse } \\
\text { accouchement et puerpéralité) / décédé }\end{array}$ \\
\hline \multicolumn{2}{|l|}{ Structures familiales } \\
\hline Taille & Nombre de membres (inférieur à 5 / égal ou supérieur à 5) \\
\hline Composition & $\begin{array}{l}\text { Caractéristiques du chef et des membres (lien avec le chef de ménage, } \\
\text { âge, sexe) }\end{array}$ \\
\hline Type & Nucléaire / élargie \\
\hline
\end{tabular}


Figure 2 : Modélisation de l'effet des migrations forcées sur la santé des populations

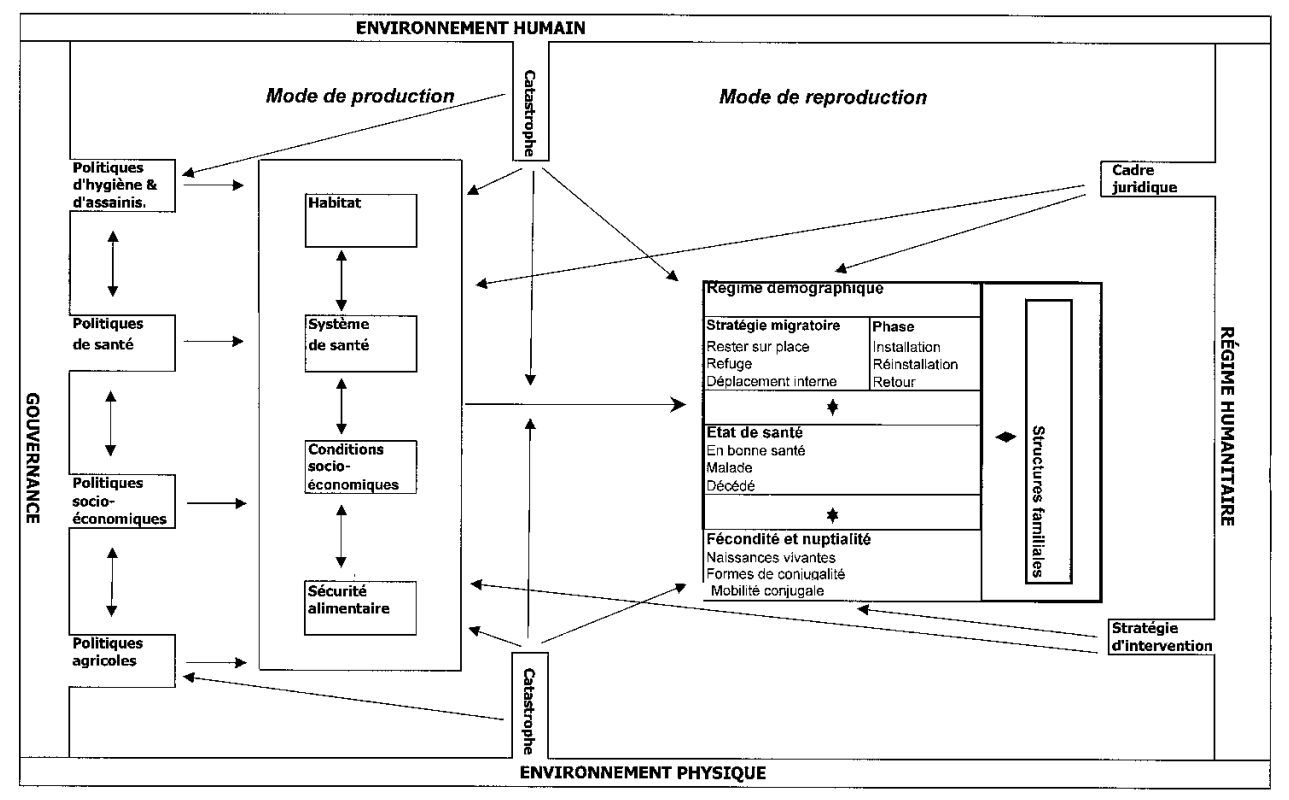

14 Les paramètres du mode de production sont perturbés par la transformation de l'environnement des populations à la suite des catastrophes. Ainsi, la destruction du système de distribution de l'eau compromet l'approvisionnement en eau potable, alors que les problèmes posés par l'évacuation des eaux usées, par le contrôle des déchets humains et des ordures affectent les conditions d'hygiène et d'assainissement. La santé est compromise par la destruction ou l'insuffisance des structures de santé et des équipements sanitaires, la réduction de la disponibilité et de l'accessibilité aux médicaments et aux transfusions sanguines sûres et aux condoms. On assiste à la détérioration de la qualité du système de santé, alors que certains programmes nationaux de prévention et de promotion de la santé sont suspendus, et que l'efficacité des systèmes de surveillance des maladies décline. Les prestataires de services subissent une surcharge de travail ou migrent à leur tour afin d'échapper à la crise. Dans le cas des conflits armés, le budget de la santé est souvent transféré vers les dépenses militaires.

\section{L'effet de la migration forcée sur l'état de santé}

Ce contexte socio-démographique et de santé est susceptible d'affecter différemment les réfugiés, les personnes déplacées et les populations restés sur place. Ainsi, il a été démontré au Tchad que les personnes déplacées constituent un groupe à risque sur le plan démographique. En particulier, le risque de divorce des chefs de ménage ayant déjà été des personnes déplacées à l'intérieur du Tchad était près de trois fois plus élevé que celui des non migrants, contrairement aux réfugiés ayant connu l'exil (Laliberté, Laplante et Piché, 2003). Les personnes restées sur place subissent le contexte général de détérioration du système de santé du pays où intervient la crise humanitaire, du fait de leur résilience ou de l'impossibilité pour elles de migrer. Quant à celles qui fuient dans une zone à l'intérieur du même pays, elles espèrent améliorer leur situation, mais leur mobilité entrave la réception d'une assistance humanitaire et rend difficile le contrôle des épidémies, surtout dans le cas de mouvements de masse à l'intérieur d'une 
vaste zone géographique. Elles constituent un groupe à risque en tant que cible et enjeu militaire. La mobilité entrave le dépistage, le traitement et le suivi des maladies et des vaccinations, tout en augmentant les risques de non-observance thérapeutique. Un mauvais usage et un manque de contrôle des protocoles médicaux peuvent engendrer une résistance aux médicaments. L'habitat souvent précaire des personnes durant leur déplacement a aussi un impact sur leur santé. Le taux brut de mortalité des personnes déplacées serait même significativement plus élevé que celui des personnes restées sur place, entre autres à cause d'un accès réduit aux services de santé, à l'eau potable, à l'hygiène et à un abri convenable, mais aussi en raison du manque d'information sur le nouvel environnement et de la perte des biens et réseaux sociaux (Bayard, 2004:5).

L'assistance à des personnes regroupées dans des camps est plus aisée que celle destinée à des réfugiés ou des personnes déplacées dispersés sur un grand territoire. Par contre, la forte densité de la population dans les camps favorise la propagation des maladies transmissibles et pose des défis sur les plans de l'hygiène et de l'assainissement. Ainsi, dans les camps de réfugiés, la mortalité des enfants âgés de moins de cinq ans semble être corrélée à la taille de la population ${ }^{9}$ (Mercer, 1992 dans Spiegel, Sheik, Gotway-Crawford et Salama, 2002: 1932). Cependant, une telle corrélation est susceptible de varier en fonction de l'évolution de la crise humanitaire, et d'être moins évidente lorsque la phase d'urgence s'est stabilisée. Les soins de santé, y compris les programmes de vaccination, sont théoriquement plus disponibles et facilement accessibles. L'état de santé des réfugiés établis dans les populations d'accueil est tributaire de leur capacité d'accès à un système local performant et en mesure de répondre à la fois à la demande des nationaux et des réfugiés. L'emplacement des camps a un effet significatif sur l'état de santé : un espace/temps trop faible entre le site et la zone de conflit augmente les risques d'exposition à la violence. Ainsi, une étude réalisée dans 51 camps de réfugiés situés dans sept pays dont trois en Afrique (Éthiopie, Tanzanie et Ouganda) a démontré que le taux brut de mortalité dans les sites situés à moins de dix kilomètres de la zone de conflit était dix fois plus élevé que pour les camps éloignés d'au moins cinquante kilomètres (Spiegel, Sheik, Gotway-Crawford et Salama, 2002 : 1927). La distance entre le camp et la structure sanitaire de référence aurait aussi un effet significatif sur le taux de mortalité. De même, le degré d'insécurité d'une zone affecte indirectement la santé en compromettant l'accès des organismes humanitaires aux populations. Les problèmes de malnutrition et d'eau potable sont susceptibles de varier selon le statut migratoire et la nature de l'insertion dans la zone d'accueil ; en effet, des difficultés d'approvisionnement risquent de se manifester dans le cas des personnes déplacées, alors que les réfugiés dans les camps ont un accès plus facile à l'eau potable, à des rations alimentaires et à plus long terme à des programmes d'autosuffisance alimentaire. Par contre, la situation des réfugiés hors camps est tributaire de la disponibilité et de l'accès aux aliments dans le pays d'accueil, et à long terme aux modalités d'insertion dans l'économie locale. L'apparition de maladies diarrhéiques est reliée à la qualité de l'eau, à l'hygiène et à la disponibilité d'équipements sanitaires adéquats dans la zone d'accueil. L'accès aux préservatifs et à des programmes de prévention, de diagnostic et de soins est plus grand pour les réfugiés dans les camps que pour les personnes déplacées ou les réfugiés hors camps. Cependant, ces migrants et les travailleurs humanitaires seraient moins réceptifs aux programmes de sensibilisation et d'éducation au VIH/sida, le risque de subir la violence ou de mourir d'autres maladies plus immédiates étant prédominant (Forced Migration Review, $2004: 24$ et Williamson, $2004: 23$ ). La nature même de la crise humanitaire joue 
un rôle déterminant sur la transmission du virus. Ainsi, les violations des droits humains, la violence et la persécution basées sur le genre augmentent durant les guerres et les conflits armés (Laliberté, 2000: 58). Paradoxalement, alors que les femmes se réfugient pour fuir la persécution et violence, le facteur de risque est précisément plus élevé durant la fuite et l'exil (UNHCR, 1995). Des femmes sont agressées sexuellement par des individus qui utilisent leur position de pouvoir dans les zones de conflit, aux frontières et dans des camps (Laliberté, 2000 : 59).

\section{Conclusion}

Les crises humanitaires amènent des individus, des familles et des communautés à migrer afin d'assurer leur survie; ces crises et les migrations qui en découlent engendrent des situations de vulnérabilité en matière de santé. On manque encore d'études fondées sur un protocole de recherche scientifique permettant de mesurer l'effet réciproque des facteurs explicatifs sur l'état de santé des divers segments des populations. Notre cadre d'analyse pourrait être opérationnalisé en quantifiant empiriquement ses diverses variables : en amont de la problématique à l'étude, les facteurs environnementaux responsables de la crise; au niveau macro-sociétal, ceux relatifs à la gouvernance, aux enjeux politiques, aux cadres politiques et au régime humanitaire qui sont susceptibles d'affecter l'état de santé. Les aspects intermédiaires reliés au mode de production doivent aussi être incorporés dans l'analyse. L'état de santé doit être considéré dans le cadre de l'ensemble du régime démographique, avec une attention particulière accordée aux structures familiales. Il est enfin essentiel de tenir compte de la dimension temporelle des conflits et de la dynamique démographique, en lien avec les diverses phases traversées par les réfugiés et personnes déplacées dans le cadre de leur migration forcée : arrivée dans l'urgence, installation dans la société de la région ou du pays d'asile, éventuellement mobilités ultérieures (réinstallation et retour).

\section{BIBLIOGRAPHIE}

BAYA Banza et LALIBERTÉ Danièle (2007) Crise des solidarités ou solidarités dans la crise ? Une lecture à partir de l'analyse de l'évolution de la structure des ménages entre 1993 et 2003 à Ouagadougou au Burkina Faso, Population et développement en Afrique : questions émergentes, 5ème Conférence africaine sur la population, Arusha, Tanzanie, Décembre 10-14, 2007.

BAYARD Robert (2004) FMO Thematic Guide : Forced Migration and Public Health, FMO Research Guide, July, 29 p.

COMITÉ INTERNATIONAL DE LA CROIX-ROUGE (2003) Droit international humanitaire et droit international des droits de l'homme. Similitudes et différences, 2 p. http://www.icrc.org 
CONNOLY Màire A., GAYER Michelle, RYAN Michael J., SALAMA Peter, SPIEGEL Paul et HEYMANN David L. (2004) Communicable diseases in complex emergencies : impact and challenges, Lancet, 364, pp. 1974-1983.

CONOIR Yvan (2002) «Évolution de la nature des opérations militaires », dans Conoir Yvan et Gérard Verna (Dir,), L'action humanitaire du Canada. Histoire, concepts, politiques et pratiques de terrain, Les Presses de l'Université Laval, 615 p., pp. 146-156.

CREEL Liz (2002) Répondre aux besoins de santé reproductive des personnes déplacées, in Rapport de politique générale, Population Reference Bureau, Measure Communication, septembre 2002, 7 p.

FNUAP, HCR et OMS (2001) La santé reproductive en situation de réfugiés, Manuel de terrain interorganisations, 129 p., http://www.who.int/reproductive-health/publications

FORCED MIGRATION REVIEW (2004) Tackling HIV AIDS in post-war Sierra Leone, compiled by the Forced Migration Review editors from submissions by CARE, IRC and ARC, Forced Migration Review 19 (January), pp. 24-25.

GREGORY Joel W. et PICHÉ Victor (1985) Mode de production et régime démographique, Revue canadienne des études africaines, 19 (1), pp. 73-79.

INTERNATIONAL COMMITTEE OF THE RED CROSS (1998) Guiding Principles on Internal Displacement, International Review of the Red Cross, 324, pp. 545-556, http://www.icrc.org

KINNAH G. (1996) An Account of a personal experience, in Refugees, Displaced People and their vulnerability to HIV/AIDS, Report on the Seminar on NGO Action, UK NGO AIDS CONSORTIUM, 28-29 October 1996, London, pp. 8-10.

LALIBERTÉ Danièle, LAPLANTE Benoît et PICHÉ Victor (2003) The impact of forced migration on marital life in Chad, European Journal of Population, 19 (4), pp. 413-435.

LALIBERTÉ-BÉRINGAR Danièle (2002) Problématique du genre dans les situations de détresse : le cas des réfugiés en Afrique, Refuge, 20 (4), pp. 53-62.

LAUTZE Sue, LEANING Jennifer, RAVEN-ROBERTS Angela, KENT Randolph et MAZURANA Dyan (2004) Assistance, protection and governance networks in complex emergencies, Lancet, 364 (December 11), pp. 2134-2141.

LE PROJET SPHÈRE (2004) Charte humanitaire et normes minimales pour les interventions lors de catastrophes, OXFAM Publishing, 412 p., http://www.sphereproject.org

M'BOUSSA J., YOKOLO D., PEREIRA B. et EBATA-MONGO S. (2002) Flambée de la tuberculose en situation de conflit armé : le cas du Congo Brazzaville, Traduction de l'article : A flare-up of tuberculosis due to war in Congo Brazzaville, Int J Tuberc Lung Dis 6 (6), pp. 475-478.

MCGINN Therese (2000) Reproductive Health of War-Affected Populations : What do we know? International Family Planning Perspectives, 26 (4) pp. 174-180.

ORACH Garimoi Orach et DE BROUWERE Vincent (2004) Post-emergency health services for refugee and host populations in Uganda, 1999-2002, Lancet, 364, pp. 611-612.

SALAMA Peter, SPIEGEL Paul, TALLEY Leisel et WALDMAN Ronald (2004) Lessons learned from complex emergencies over past decade, Lancet, 364 (November 13), pp. 1801-1813.

SPIEGEL Paul, SHEIK Mani, GOTWAY-CRAWFORD Carol et SALAMA Peter (2002) Health Programmes and policies associated with decreased mortality in displaced people in postemergency phase camps : a retrospective study, Lancet, 360 (December 14), pp. 1927-1934. 
ST-PIERRE Monique (2002) «L'ingérence humanitaire : une brève histoire », dans Conoir Yvan et Gérard Verna (Dir,), L'action humanitaire du Canada. Histoire, concepts, politiques et pratiques de terrain, Les Presses de l'Université Laval, 615 p., pp. 45-58.

TABUTIN Dominique et BARTIAUX Françoise (1986) Structures familiales et structures sociales dans le Tiers-Monde, in AIDELF (édit.) Les familles d'aujourd'hui, Colloque no. 2, Genève, 17-20 septembre 1984, PUF, Paris, pp. 231-243.

UNHCR (2007) 2006 Global Refugee Trends.Refugees, Asylum-seekers, Returnees, Internally Displaced and Stateless Persons, Division of Operational Services, Field Information and Coordination Support Division, June 2007, Revised 16 July 2007, http://www.unhcr.org

UNHCR (1995) The State of the World's Refugees in search of solutions, Oxford University Press, http:// www.unhcr.org/publ

VAN DAMME Wim, DE BROUWERE Vincent, BOELAERT Marleen et VAN LERBERGHE Wim (1998) Effects of a refugee-assistance programme on host population in Guinea as measured by obstetric interventions, The Lancet, 351 (May 30), pp. 1609-1613.

WILLIAMSON Katharine (2004) AIDS, Gender and Refugee Protection Framework, Refugee Study Center Working Paper 19, Working Paper Series, Queen Elizabeth House, International Development Centre, University of Oxford, $36 \mathrm{p}$.

\section{NOTES}

1. Selon les critères internationaux, une situation est qualifiée de crise humanitaire quand le taux brut de mortalité atteint un décès ou plus par jour pour 10000 personnes, ou que le taux de mortalité infanto-juvénile quotidien dépasse 2 décès par 10000.

2. Étant par ailleurs entendu que des dispositions relevant du Droit international humanitaire s'appliquent aussi aux réfugiés.

3. En autres l'article 25 de la Déclaration universelle des droits de l'homme (1948), l'article $12 \mathrm{du}$ Pacte international relatif aux droits économiques, sociaux et culturels (1966), et le Pacte international relatif aux droits civils et politiques (1966).

4. Plus particulièrement les articles $20,21,23$ et 24 de la Convention des Nations unies pour les Réfugiés (1951).

5. Les quatre Conventions de Genève de 1949 et leurs deux protocoles de 1977 couvrent la santé des réfugiés et personnes déplacées dans leur pays.

6. En autres par la Convention sur l'élimination de toutes les formes de discriminations à l'égard des femmes (1979), la Convention relative aux droits de l'enfant (1989) et la Charte africaine des droits de l'homme et des peuples (1981).

7. Force de travail humaine et moyens de production tels que outils, équipements, technologies, infrastructure et terre.

8. Les relations de production réfèrent aux structures sociales qui régulent la relation entre les humains et la production des biens. Citons la propriété, le pouvoir, les relations entre les classes sociales, les relations de travail coopératives et associatives.

9. Par ailleurs, la taille de la population des camps ne doit pas dépasser 50000 personnes selon les normes internationales (Le Projet Sphère, 2004). 


\section{AUTEUR}

DANIĖLE LALIBERTÉ

Chercheure associée au Centre interuniversitaire d'études démographiques. Département de

Démographie. Université de Montréal. daniele.laliberte@umontreal.ca 